\title{
Polarization-Driven Edge-State Transport in Transition-Metal Dichalcogenides
}

Ke Wang ${ }^{1, \dagger}$, Gongwei $\mathrm{Hu}^{1, \dagger}$, Ruhao $\mathrm{Liu}^{1}$, Yaming Zhang ${ }^{1}$, Minjiang Dan ${ }^{1}$, Lijie $\mathrm{Li}^{2}$, and Yan Zhang $^{1,3,4, *}$

${ }^{1}$ School of Physics, University of Electronic Science and Technology of China, Chengdu 610054, China

${ }^{2}$ Multidisciplinary Nanotechnology Centre, College of Engineering, Swansea University, Swansea, SA1 8EN, UK

${ }^{3}$ CAS Center for Excellence in Nanoscience, Beijing Institute of Nanoenergy and Nanosystems, Chinese Academy of Sciences; National Center for Nanoscience and Technology (NCNST), Beijing 100083, China

${ }^{4}$ School of Nanoscience and Technology, University of Chinese Academy of Sciences, Beijing 100049, P. R. China

†Those authors contribute equally to this work.

*To whom correspondence should be addressed, E-mail: zhangyan@uestc.edu.cn

\begin{abstract}
Intrinsic polarization has been demonstrated in layered structures to reduce the energy gap. Here we demonstrate that strain-induced polarization can increase energy gap and induce a metallic-to-semiconducting phase transition in zigzag nanoribbons of single-layered transitionmetal dichalcogenides, such as $\mathrm{MoS}_{2}, \mathrm{MoSe}_{2}, \mathrm{WS}_{2}$ and $\mathrm{WSe}_{2}$. This study provides a guidance for designing quantum piezotronic devices.
\end{abstract}

PACS numbers: 73.20.At, 73.43. Nq, 77.22.Ej 


\section{INTRODUCTION}

Two-dimensional (2D) materials have been attracting more and more attentions in fundamental researches [1-3] and device applications [4, 5], such as graphene and monolayer transition-metal dichalcogenides (TMDs) [6, 7]. TMDs have strong spin-orbit coupling, large band gap and boundary metallicity compared with gapless graphene [8-10]. By manipulating edge states, such as strain engineering [11], applying electric field [12] and external exchange field [13], electronic properties of TMDs nanoribbons can be half-metallic [13], magnetic [14] and superconductive [15]. Topological superconductivity and Majorna zero-energy modes have been demonstrated based on edge states of monolayer $\operatorname{MoS}_{2}$ [15]. Edge states can be used for $\mathrm{MoS}_{2}$ field effect transistors [16], and a wideband Q-switching fiber laser [17].

Strain-induced polarization provides an effective method to tune/control carrier transport properties without changing the interfacial structure or chemistry [18]. Based on strain-induced polarization, piezotronics and piezo-phototronics are two emerging fields in nanotechnology for sensors, actuators, energy-harvesting and active optoelectronic devices [18, 19]. Polarization can drive a topological insulator transition in $\mathrm{GaN} / \mathrm{InN} / \mathrm{GaN}$ and $\mathrm{GaAs} / \mathrm{Ge} / \mathrm{GaAs}$ quantum wells $[20,21]$. The mechanical properties and layered structures allow 2D piezoelectric materials to overcome bulk piezoelectric material flexibility limitations. Monolayer $\mathrm{MoS}_{2}$ exhibits piezoelectric property due to the broken center inversion symmetry $[22,23]$, allowing monolayer $\mathrm{MoS}_{2}$ to be applied in low-dimensional electro-mechanical devices [24-27]. Strain-induced polarization on two-dimensional materials have been applied for self-powered electro-mechanical devices [24, 28], such as nanogenerators [24], strain sensors [29] and field effect transistors [26, 27]. $\mathrm{MoS}_{2}$ zigzag nanoribbons showed the metallic edge states [30]. A transverse electric field can drive the electrons to transfer between the Moand S- terminated boundary, resulting in the edge states phase transition [13]. Whether polarization can increase energy gap for edge states transition remains an open question.

\section{MODELLING AND RESULTS}

In this study, we theoretically study polarization-driven edge-state transport of single-layer zigzag nanoribbons of $\mathrm{MoS}_{2}, \mathrm{MoSe}_{2}, \mathrm{WS}_{2}$ and $\mathrm{WSe}_{2}$. Strain-induced polarization can increase edge-state band gap, and result in a phase transition from metallic to semiconducting. Single- 
layer TMD consists of one transition metal layer sandwiched between two chalcogen layers with the form $\mathrm{MX}_{2}$, where $\mathrm{M}$ is the transition metal (Mo, W...), and $\mathrm{X}$ is the chalcogen ( $\mathrm{S}$, Se...). Schematic diagram of $\mathrm{MoS}_{2}$ nanoribbon is sketched in Figure 1(a).

An 11-band Hamiltonian has been widely used for the basic electronic properties of singlelayered $\mathrm{MoS}_{2}$ [31]. By performing a unitary transformation based on horizontal reflection symmetry, the Hamiltonian can be described in the reduced atomic orbital basis as [32]

$$
\left[d_{x^{2}-y^{2}}, d_{x y}, d_{3 z^{2}-r^{2}}, \frac{1}{\sqrt{2}}\left(p_{x}^{t}+p_{x}^{b}\right), \frac{1}{\sqrt{2}}\left(p_{y}^{t}+p_{y}^{b}\right), \frac{1}{\sqrt{2}}\left(p_{z}^{t}-p_{z}^{b}\right)\right]
$$

where $t$ and $b$ stand for top and bottom Sulfur layers respectively. Then the Hamiltonian is given by [13]

$$
\begin{aligned}
H= & \sum_{i, \mu}\left(\varepsilon_{i, \mu}^{M} c_{i, \mu}^{\dagger} c_{i, \mu}+\varepsilon_{i, \mu}^{S} b_{i, \mu}^{\dagger} b_{i, \mu}\right)+\sum_{\langle\langle i j\rangle\rangle, \mu v}\left(t_{i j, \mu v}^{M M} c_{i, \mu}^{\dagger} c_{j, v}+t_{i j, \mu v}^{S S} b_{i, \mu}^{\dagger} b_{j, v}\right) \\
& +\sum_{\langle i j\rangle, \mu v}\left(t_{i j, \mu \nu}^{M S} c_{i, \mu}^{\dagger} b_{j, v}\right)+\text { H.c. }
\end{aligned}
$$

where $i, j$ and $u, v$ stand for lattice sites and atomic orbital indices, respectively, $\varepsilon_{i}^{M}$ and $\varepsilon_{i}^{S}$ represent onsite energies for Mo and S atoms, $c_{i, \mu}^{\dagger}\left(b_{i, \mu}^{\dagger}\right)$ is creation operator for Mo (S) and $c_{i, \mu}\left(b_{i, \mu}\right)$ is the corresponding annihilation operator, $t_{i j, \mu v}^{M M}, t_{i j, \mu v}^{S S}$, and $t_{i j, \mu v}^{M S}$ respectively represent hopping energy between Mo-Mo, S-S and Mo-S atoms with a matrix form.

We simultaneously consider the effects of deformation potential and polarization on transport properties in this model when an external strain is applied. In the presence of a tensile strain, $\mathrm{MoS}_{2}$ unit cell becomes stretched along strain direction while the unit cell is compressed under compressive strain, as illustrated in Figure 1(a). Polarization is induced by separating the center of transition metal atoms (with positive charge) and chalcogenide atoms (with negative charge), leading to a polarization inside the material.

In our model, strain-induced polarization is included in the onsite energies, which are written as [13], 


$$
\begin{aligned}
\varepsilon_{i}^{M} & =\left[\begin{array}{ccc}
\Delta_{0} & 0 & 0 \\
0 & \Delta_{2} & -i \lambda_{M} s_{z} \\
0 & i \lambda_{M} s_{z} & \Delta_{2}
\end{array}\right]+V_{\text {piezo }} \mathbf{I} \\
\varepsilon_{i}^{S} & =\left[\begin{array}{ccc}
\Delta_{p}+t_{x x}^{\perp} & -i \lambda_{S} s_{z} / 2 & 0 \\
i \lambda_{S} s_{z} / 2 & \Delta_{p}+t_{y y}^{\perp} & 0 \\
0 & 0 & \Delta_{z}-t_{z z}^{\perp}
\end{array}\right]+V_{\text {piezo }} \mathbf{I}
\end{aligned}
$$

where $i$ stands for lattice site, $\lambda_{M}=0.075 \mathrm{eV}$ and $\lambda_{S}=0.052 \mathrm{eV}$ are the spin-orbit coupling of Mo and S atoms, respectively, $s_{z}$ represents $z$-component spin degree of freedom, $V_{\text {piezo }}$ is the piezoelectric potential at the site, $\mathbf{I}$ is identity matrix and $t_{x x}^{\perp}, t_{y y}^{\perp}$ and $t_{z z}^{\perp}$ indicate the interlayer hopping between two Sulfur layers, $\Delta_{0}, \Delta_{2}, \Delta_{p}$ and $\Delta_{z}$ are the crystal field of atomic level at the $l=0\left(d_{3 z^{2}-r^{2}}\right), \quad l=2\left(d_{x^{2}-y^{2}}, d_{x y}\right)$ Mo orbitals, the in-plane $\left(p_{x}, p_{y}\right)$ and the out-of-plane $p_{z} \mathrm{~S}$ orbitals, respectively. In our study the spin-orbit coupling is ignored as we only focus on the transport properties of the nanoribbons under strain. Other Slater-Koster tight-binding parameters are cited from Ref [33]. The hopping terms $t_{i j, \mu v}^{M M}$, $t_{i j, \mu v}^{S S}$, and $t_{i j, \mu v}^{M S}$ are detailed in Ref. [31,33,34].

Similar to previous works $[34,35]$, the effect of strain-induced deformation potential in our model is included by modifying the hopping energies as,

$$
t_{i j, \mu v}=t_{i j, \mu v}^{0}\left(1-\beta_{i j, \mu v} \frac{\left|\mathbf{R}_{i j}-\mathbf{R}_{i j}^{0}\right|}{\left|\mathbf{R}_{i j}^{0}\right|}\right)
$$

where $i, j$ and $u, v$ stand for lattice sites and atomic orbital indices, respectively, $\mathbf{R}_{i j}^{0}$ is the equilibrium position vector of $i$ - and $j$-th atoms, $\beta_{i j, \mu v}$ is the electron-phonon coupling coefficient, $\mathbf{R}_{i j}$ is the modified position vector under strain. The electron-phonon coupling $\beta_{i j, \mu v}$ is respectively estimated to be 3, 4 and 5 for $p p, p d$ and $d d$ orbital hybridizations [34]. Under homogenous strain, $\mathbf{R}_{i j}$ can be obtained by $\mathbf{R}_{i j}=\mathbf{R}_{i j}^{0} \cdot(1+\mathbf{s})$, where $\mathbf{S}$ is the strain tensor. 
Hexagonal structured $\mathrm{MoS}_{2}$ has only one independent piezoelectric coefficient $e_{11}$ due to its $D_{3 h}$ point group [24], and the other non-zero piezoelectric coefficients can be described as $e_{11}=-e_{12}=-e_{26} / 2$ [36]. Therefore, for an axial strain, polarization is induced only along armchair direction and can be expressed as $P_{1}=e_{11} s_{11}+e_{12} s_{22}=e_{11}\left(s_{11}-s_{22}\right)$ [24]. When a uniform strain $S_{11}$ is applied along $y$ direction (armchair direction), strain-induced polarization and electric potential can be given from an infinite long charged wire model as follows [37-39]

$$
\begin{gathered}
E_{\text {ріего }}=\frac{e_{11} s_{11}}{2 \pi \varepsilon_{r} \varepsilon_{0}}\left(\frac{1}{y+L / 2}+\frac{1}{L / 2-y}\right) \\
V_{\text {ріеzо }}=\frac{e_{11} s_{11}}{2 \pi \varepsilon_{r} \varepsilon_{0}} \ln \left|\frac{y+L / 2}{L / 2-y}\right|
\end{gathered}
$$

where $e_{11}$ is the piezoelectric coefficient along $y$ direction, $\varepsilon_{r}$ is the relative dielectric constant, $\varepsilon_{0}$ is the vacuum dielectric constant, $L$ is the nanoribbon width. Piezoelectric coefficients of four TMDs are cited from Ref. [23]. Figure 1(b) calculates polarization in a single-layer $\mathrm{MoS}_{2}$ nanoribbon with the width of $4.1 \mathrm{~nm}$ under $2 \%$ strain. The polarization reaches the order of $\mathrm{MV} / \mathrm{cm}$ at two boundaries.

Based on tight-binding model, we use open source software package KWANT to calculate quantum transport [40]. Figure 2(a) shows band structure of unstrained zigzag $\mathrm{MoS}_{2}$ nanoribbon, and ribbon width is set to be $4.1 \mathrm{~nm}$. The conduction minimum $(\mathrm{CM})$ and valence maximum (VM) respectively correspond to S-terminated edge state and Mo-terminated edge state [13]. The band gap is negative with the CM lower than the VM, leading to metallic phase of edge states. With increasing external strain, the negative band gap starts to increase and then becomes zero at a critical strain 2.7\%, as shown in Figure 2(b). Increasing strain to 5\%, edge states have a band gap of $0.34 \mathrm{eV}$, as shown in Figure 2(c). Positive potential of S boundary raises the $\mathrm{CM}$, and the $\mathrm{VB}$ is reduced by negative potential of Mo boundary, giving rise to the phase transition from metallicity (negative band gap) to semiconductor (positive band gap). This behavior has also been observed in armchair $\mathrm{MoS}_{2}$ nanoribbon by applying an electric 
field [41].

When the ribbon accounts for spin-orbit interaction (SOI), the band structure shows a slight spin splitting for edge states, as shown in Fig. 2(d)-2(f). The effect of strain-induced polarization on edge state is the same as the case without SOI in Figures. 2(a)-2(c) whereas only a slight spin splitting appears. The spin subbands are separated along momentum axis, leading to the spin-dependent electronic transport.

According to Ref. [13], strong external electric field can induce a peculiar half-metallic state for zigzag $\mathrm{MoS}_{2}$ ribbon when accounting for SOI and exchange field. Actually, straininduced polarization field can also trigger such half-metallic state at a proper strain. For instance, in a $4.1 \mathrm{~nm} \mathrm{MoS}$ ribbon with an exchange coupling strength $h=\lambda_{M}$ and intrinsic SOI, strain $s_{11}=4 \%$ can induce a half-metallic state. APPENDIX A shows that although the ribbon width and exchange field are smaller than those in Ref. [13], strain-induced polarization can also trigger the half-metallic state. Half-metallic state can be used to design spin-related devices, such as spin filtering devices [13]. Therefore, strain-induced polarization is an effective approach to design spintronic devices based on the edge states of zigzag nanoribbon.

Edge-state transport properties of zigzag $\mathrm{MoS}_{2}$ are explored in the presence of strain. Figure 3(a) shows the conductance versus electronic energy under various strains. Here, conductance unit is $G_{0}=e^{2} / h$ ( $e$ is electron charge and $h$ is Planck constant). In the insert of Figure 3(a), the gradient color signifies the potential distribution, and the direction of polarization $\mathrm{E}_{\text {piezo }}$ is reverse to $y$ axis. There is a $4 G_{0}$ conductance range without strain, owing to an overlap of electronic energies for two edge states. Under the gap closing threshold strain (2.7\%), the conductance remains a constant over the entire edge-state region with the conductance of $2 G_{0}$. The nanoribbon becomes semiconducting at $5 \%$ strain. There is a zero conductance range where electronic energy is located on the gap.

Figure 3(b) shows edge-state band gap versus external strain for four TMDs. Without strain, $\mathrm{MoS}_{2}, \mathrm{MoSe}_{2}, \mathrm{WS}_{2}$ and $\mathrm{WSe}_{2}$ have a negative band gap of $-0.38 \mathrm{eV},-0.59 \mathrm{eV},-0.11 \mathrm{eV}$ and $-1.19 \mathrm{eV}$, respectively. Band gap approximately linearly increases with the strain. When the strain increases to $8 \%$, the band gap of $\mathrm{MoS}_{2}, \mathrm{MoSe}_{2}$ and $\mathrm{WS}_{2}$ becomes a positive value of 0.78 
$\mathrm{eV}, 0.34 \mathrm{eV}$, and $0.22 \mathrm{eV}$ respectively. $\mathrm{WSe}_{2}$ still has a negative gap of $-0.37 \mathrm{eV}$. The different gap behavior of $\mathrm{WSe}_{2}$ is due to its wider inverted gap and weaker piezoelectricity. $\mathrm{WSe}_{2}$ has a negative gap -1.19 eV, much lower than three other materials. For opening such large negative gap, a high piezoelectric potential is required. From Eq. (7), piezoelectric potential is dependent on the piezoelectric coefficient divided by the relative permittivity $e_{11} / \varepsilon_{r}$ that is $\sim 12 \mathrm{pC} / \mathrm{m}$ for $\mathrm{WSe}_{2}$, lower than $\mathrm{MoS}_{2}, \mathrm{MoSe}_{2}$ and $\mathrm{WS}_{2}(18,16$ and $15 \mathrm{pC} / \mathrm{m})$. This indicates that at a fixed external strain, $\mathrm{WSe}_{2}$ has smaller piezoelectric potential in comparison with other three materials.

For a wider ribbon such as $10 \mathrm{~nm}$ and $50 \mathrm{~nm}$, the edge state still exists and its gap also shows a linear increase with the applied strain, similar to the case of $4.1 \mathrm{~nm}$ width ribbon. APPENDIX B shows that the critical strain decreases with the ribbon width and being $2.07 \%$ and $1.37 \%$ for the ribbon width of $10 \mathrm{~nm}$ and $50 \mathrm{~nm}$, respectively.

We calculate the conductance in Figures 4(a) - 4(d) for $\mathrm{MoS}_{2}, \mathrm{MoSe}_{2}, \mathrm{WS}_{2}$ and $\mathrm{WSe}_{2}$ respectively. Dashed lines signify the change of two edge states (M-terminated and Xterminated boundary). The results show that for four different materials, strain increases Mterminated edge-state energy and decreases S-terminated edge-state energy. There is an intersection indicating the opening of band gap. For $\mathrm{MoS}_{2}, \mathrm{MoSe}_{2}, \mathrm{WS}_{2}$ and $\mathrm{WSe}_{2}$, the critical strains to open the band gap are $2.7 \%, 5.2 \%, 3.8 \%$ and $11.5 \%$, respectively. Bulk states lead to higher conductance which is shown in the red region in Figure 4.

Different from the situation in zigzag nanoribbons, armchair nanoribbons have an intrinsic edge-state band gap. There are many differences between zigzag and armchair nanoribbons. Zigzag nanoribbon has two separated edge state (Mo edge and S edge) which are degenerate in armchair nanoribbon [12]. These degenerated edge states can be separated by a $y$-axis electric field $[12,42]$. Moreover, the modulation mechanism of electronic transport is also quite different for these two forms of nanoribbons. To be more explicit, polarization ( $y$ axis) is perpendicular to the transport direction ( $x$ axis) for zigzag nanoribbon, as shown in the insert of Figure 3(a). For armchair nanoribbon, the polarization is parallel to the transport direction [43].

\section{CONCLUSION}


To summarize, we theoretically investigate polarization-driven edge-state transport in zigzag TMDs nanoribbons. Edge-state band gap changes from a negative to positive value with the increase of the externally applied strain, indicating a phase transition from metallicity to semiconducting. This study provides a guidance for designing high-performance piezotronic devices based on strain modulated quantum states. 


\begin{abstract}
APPENDIX A: HALF-METALLIC STATE
Figure 5 shows band structure of the $\mathrm{MoS}_{2}$ ribbon at strain $s_{11}=4 \%$ when SOI and exchange coupling are simultaneously taken into account. Exchange coupling is set at the strength $h=\lambda_{M}$, which is lower than $h=2.4 \lambda_{M}$ in Ref. [13]. In spite of a lower exchange coupling, strain-induced polarization can induce a peculiar half-metallic state, as shown in Figure 5. We note that despite different ribbon width $(4.1 \mathrm{~nm}$ in this study and $6.4 \mathrm{~nm}$ in Ref. [13]), the edge-state subbands can also touch each other, similar to Figure 2(a) of Ref. [13], where exchange coupling is fixed at $h=2.4 \lambda_{M}$.
\end{abstract}

\title{
APPENDIX B: INFLUENCE OF WIDTH ON GAP OPENING BEHAVIOR
}

Due to the width dependence of piezoelectric potential (Eq. (7)), the edge-state gap behavior is associated with the ribbon size. Figure 6 calculates the edge-state gap as a function of strain for three widths, namely 4.1, 10 and $50 \mathrm{~nm}$. The monolayer $\mathrm{MoS}_{2}$ with those widths can be synthesized in experiment [44-46]. It shows that regardless of ribbon width, the gap always increases linearly with external strain. Increasing ribbon width can decrease the critical strain for zero gap, which is due to the rising piezoelectric potential with width. The critical strains are $2.72 \%, 2.07 \%$ and $1.37 \%$ for the ribbon width of $4.1,10$ and $50 \mathrm{~nm}$, respectively. 


\section{Reference}

[1] C.L. Kane, E.J. Mele, Quantum spin Hall effect in graphene, Physical Review Letters, 95 (2005) 226801.

[2] D. Xiao, G.-B. Liu, W. Feng, X. Xu, W. Yao, Coupled spin and valley physics in monolayers of MoS 2 and other group-VI dichalcogenides, Physical Review Letters, 108 (2012) 196802.

[3] X. Qian, J. Liu, L. Fu, J. Li, Quantum spin Hall effect in two-dimensional transition metal dichalcogenides, Science, 346 (2014) 1344-1347.

[4] D. Jariwala, V.K. Sangwan, L.J. Lauhon, T.J. Marks, M.C. Hersam, Emerging Device Applications for Semiconducting Two-Dimensional Transition Metal Dichalcogenides, ACS Nano, 8 (2014) 1102-1120.

[5] W. Han, R.K. Kawakami, M. Gmitra, J. Fabian, Graphene spintronics, Nature Nanotechnology, 9 (2014) 794. [6] S. Manzeli, D. Ovchinnikov, D. Pasquier, O.V. Yazyev, A. Kis, 2D transition metal dichalcogenides, Nature Reviews Materials, 2 (2017) 17033.

[7] K.F. Mak, C. Lee, J. Hone, J. Shan, T.F. Heinz, Atomically thin MoS 2: a new direct-gap semiconductor, Physical Review Letters, 105 (2010) 136805.

[8] M. Bollinger, J. Lauritsen, K.W. Jacobsen, J.K. Nørskov, S. Helveg, F. Besenbacher, One-dimensional metallic edge states in MoS 2, Physical Review Letters, 87 (2001) 196803.

[9] M. Chhowalla, H.S. Shin, G. Eda, L.-J. Li, K.P. Loh, H. Zhang, The chemistry of two-dimensional layered transition metal dichalcogenide nanosheets, Nature Chemistry, 5 (2013) 263.

[10] Q.H. Wang, K. Kalantar-Zadeh, A. Kis, J.N. Coleman, M.S. Strano, Electronics and optoelectronics of twodimensional transition metal dichalcogenides, Nature Nanotechnology, 7 (2012) 699.

[11] L. Kou, C. Tang, Y. Zhang, T. Heine, C. Chen, T. Frauenheim, Tuning magnetism and electronic phase transitions by strain and electric field in zigzag MoS2 nanoribbons, The Journal of Physical Chemistry Letters, 3 (2012) 2934-2941.

[12] K. Dolui, C.D. Pemmaraju, S. Sanvito, Electric field effects on armchair MoS2 nanoribbons, ACS Nano, 6 (2012) 4823-4834.

[13] F. Khoeini, K. Shakouri, F. Peeters, Peculiar half-metallic state in zigzag nanoribbons of MoS 2: Spin filtering, Physical Review B, 94 (2016) 125412.

[14] F. Ouyang, Z. Yang, X. Ni, N. Wu, Y. Chen, X. Xiong, Hydrogenation-induced edge magnetization in armchair MoS2 nanoribbon and electric field effects, Applied Physics Letters, 104 (2014) 071901.

[15] N.F. Yuan, K.F. Mak, K. Law, Possible topological superconducting phases of MoS 2, Physical Review Letters, 113 (2014) 097001.

[16] D. Wu, X. Li, L. Luan, X. Wu, W. Li, M.N. Yogeesh, R. Ghosh, Z. Chu, D. Akinwande, Q. Niu, Uncovering edge states and electrical inhomogeneity in MoS2 field-effect transistors, Proceedings of the National Academy of Sciences, 113 (2016) 8583-8588.

[17] R.I. Woodward, E.J.R. Kelleher, R.C.T. Howe, G. Hu, F. Torrisi, T. Hasan, S.V. Popov, J.R. Taylor, Tunable Q-switched fiber laser based on saturable edge-state absorption in few-layer molybdenum disulfide (MoS2), Opt. Express, 22 (2014) 31113-31122.

[18] W. Wu, Z.L. Wang, Piezotronics and piezo-phototronics for adaptive electronics and optoelectronics, Nature Reviews Materials, 1 (2016) 16031.

[19] Z.L. Wang, W. Wu, C. Falconi, Piezotronics and piezo-phototronics with third-generation semiconductors, MRS Bulletin, 43 (2018) 922-927.

[20] M. Miao, Q. Yan, C. Van de Walle, W. Lou, L. Li, K. Chang, Polarization-driven topological insulator transition in a GaN/InN/GaN quantum well, Physical review letters, 109 (2012) 186803.

[21] D. Zhang, W. Lou, M. Miao, S.-c. Zhang, K. Chang, Interface-induced topological insulator transition in GaAs/Ge/GaAs quantum wells, Physical review letters, 111 (2013) 156402. 
[22] M.N. Blonsky, H.L. Zhuang, A.K. Singh, R.G. Hennig, Ab initio prediction of piezoelectricity in twodimensional materials, ACS Nano, 9 (2015) 9885-9891.

[23] K.-A.N. Duerloo, M.T. Ong, E.J. Reed, Intrinsic piezoelectricity in two-dimensional materials, The Journal of Physical Chemistry Letters, 3 (2012) 2871-2876.

[24] W. Wu, L. Wang, Y. Li, F. Zhang, L. Lin, S. Niu, D. Chenet, X. Zhang, Y. Hao, T.F. Heinz, Piezoelectricity of single-atomic-layer MoS 2 for energy conversion and piezotronics, Nature, 514 (2014) 470.

[25] M.-Y. Tsai, A. Tarasov, Z.R. Hesabi, H. Taghinejad, P.M. Campbell, C.A. Joiner, A. Adibi, E.M. Vogel, Flexible MoS2 field-effect transistors for gate-tunable piezoresistive strain sensors, ACS Applied Materials \& Interfaces, 7 (2015) 12850-12855.

[26] W. Bao, X. Cai, D. Kim, K. Sridhara, M.S. Fuhrer, High mobility ambipolar MoS2 field-effect transistors: Substrate and dielectric effects, Applied Physics Letters, 102 (2013) 042104.

[27] G.-H. Lee, Y.-J. Yu, X. Cui, N. Petrone, C.-H. Lee, M.S. Choi, D.-Y. Lee, C. Lee, W.J. Yoo, K. Watanabe, Flexible and transparent MoS2 field-effect transistors on hexagonal boron nitride-graphene heterostructures, ACS Nano, 7 (2013) 7931-7936.

[28] H. Zhu, Y. Wang, J. Xiao, M. Liu, S. Xiong, Z.J. Wong, Z. Ye, Y. Ye, X. Yin, X. Zhang, Observation of piezoelectricity in free-standing monolayer MoS2, Nature Nanotechnology, 10 (2014) 151.

[29] J. Qi, Y.-W. Lan, A.Z. Stieg, J.-H. Chen, Y.-L. Zhong, L.-J. Li, C.-D. Chen, Y. Zhang, K.L. Wang,

Piezoelectric effect in chemical vapour deposition-grown atomic-monolayer triangular molybdenum disulfide piezotronics, Nature Communications, 6 (2015) 7430.

[30] C. Zhang, A. Johnson, C.-L. Hsu, L.-J. Li, C.-K. Shih, Direct Imaging of Band Profile in Single Layer MoS2 on Graphite: Quasiparticle Energy Gap, Metallic Edge States, and Edge Band Bending, Nano Letters, 14 (2014) 2443-2447.

[31] E. Cappelluti, R. Roldán, J. Silva-Guillén, P. Ordejón, F. Guinea, Tight-binding model and direct-gap/indirectgap transition in single-layer and multilayer MoS 2, Physical Review B, 88 (2013) 075409.

[32] H. Rostami, R. Asgari, Valley Zeeman effect and spin-valley polarized conductance in monolayer MoS 2 in a perpendicular magnetic field, Physical Review B, 91 (2015) 075433.

[33] J.Á. Silva-Guillén, P. San-Jose, R. Roldán, Electronic Band Structure of Transition Metal Dichalcogenides from Ab Initio and Slater - Koster Tight-Binding Model, Applied Sciences, 6 (2016) 284.

[34] H. Rostami, R. Roldán, E. Cappelluti, R. Asgari, F. Guinea, Theory of strain in single-layer transition metal dichalcogenides, Physical Review B, 92 (2015) 195402.

[35] A. Castellanos-Gomez, R. Roldán, E. Cappelluti, M. Buscema, F. Guinea, H.S. van der Zant, G.A. Steele, Local strain engineering in atomically thin MoS2, Nano Letters, 13 (2013) 5361-5366.

[36] J.F. Nye, Physical properties of crystals: their representation by tensors and matrices, Oxford university press 1985.

[37] M.T. Ong, K.-A.N. Duerloo, E.J. Reed, The effect of hydrogen and fluorine coadsorption on the piezoelectric properties of graphene, The Journal of Physical Chemistry C, 117 (2013) 3615-3620.

[38] J. Zhang, W. Xie, J. Zhao, S. Zhang, Band alignment of two-dimensional lateral heterostructures, 2D Materials, 4 (2016) 015038.

[39] A. Bussy, G. Pizzi, M. Gibertini, Strain-induced polar discontinuities in two-dimensional materials from combined first-principles and Schrödinger-Poisson simulations, Physical Review B, 96 (2017) 165438.

[40] G.A. Maugin, J.D. Achenbach, Continuum Mechanics of Electromagnetic Solids, Elsevier Science2013.

[41] K. Dolui, C.D. Pemmaraju, S. Sanvito, Electric field effects on armchair MoS2 nanoribbons, ACS Nano, 6 (2012) 4823-4834.

[42] Q. Yue, S. Chang, J. Kang, X. Zhang, Z. Shao, S. Qin, J. Li, Bandgap tuning in armchair MoS2 nanoribbon, 
Journal of Physics: Condensed Matter, 24 (2012) 335501.

[43] G. Hu, L. Li, Y. Zhang, Two-dimensional electron gas in piezotronic devices, Nano Energy, 59 (2019) 667 673.

[44] J.V. Lauritsen, M. Nyberg, R.T. Vang, M.V. Bollinger, B.S. Clausen, H. Topsoe, K.W. Jacobsen, E. Laegsgaard, J.K. Norskov, F. Besenbacher, Chemistry of one-dimensional metallic edge states in MoS2 nanoclusters, Nanotechnology, 14 (2003) 385-389.

[45] A. Nourbakhsh, A. Zubair, R.N. Sajjad, K.G.A. Tavakkoli, W. Chen, S. Fang, X. Ling, J. Kong, M.S.

Dresselhaus, E. Kaxiras, K.K. Berggren, D. Antoniadis, T. Palacios, MoS2 Field-Effect Transistor with Sub-10 nm Channel Length, Nano Lett, 16 (2016) 7798-7806.

[46] X. Zhang, Z. Lai, C. Tan, H. Zhang, Solution-Processed Two-Dimensional MoS2 Nanosheets: Preparation, Hybridization, and Applications, Angewandte Chemie, 55 (2016) 8816-8838. 


\section{Figure caption}

Figure 1. (a) Schematic diagrams of zigzag $\mathrm{MoS}_{2}$ nanoribbon and $\mathrm{MoS}_{2}$ unit cell without strain, with tensile strain and compressive strain, respectively. External strain is applied in the armchair (horizontal) direction. Positive and negative signs represent positive and negative charge centers, respectively. Yellow and black atoms represent Sulfur and Molybdenum atoms, respectively. (b) The electric potential and piezoelectric field (polarization) for $\mathrm{MoS}_{2}$ nanoribbon under $2 \%$ external strain. Here, the ribbon width $\mathrm{L}$ is set at $4.1 \mathrm{~nm}$.

Figure 2. Band structures of zigzag $\operatorname{MoS}_{2}$ nanoribbon (a, d) without strain, (b, e) with 2.7\% strain and (c, f) with 5\% strain. The edge channels are marked in (a). The intrinsic spin-orbit interaction is ignored in (a)-(c) but included in (d)-(f).

Figure 3. (a) The conductance of zigzag $\mathrm{MoS}_{2}$ nanoribbon under $0 \%, 2.7 \%$ and $5 \%$ strain, respectively. The insert shows the direction of the polarization (reversely along y axis), and the gradient ramp of the insert shows the distribution of the electric potential. (b) Variation of edgestate band gap with external strain for zigzag nanoribbon of four TMDs. The dashed horizontal line indicates the zero value of band gap.

Figure 4. The conductance versus electronic energy and strain for zigzag nanoribbons of four TMDs, (a) $\mathrm{MoS}_{2}$, (b) $\mathrm{MoSe}_{2}$, (c) $\mathrm{WS}_{2}$ and (d) $\mathrm{WSe}_{2}$. The edge states and the gap opening point (GOP) are marked in each figure.

Figure 5. Band structure of half-metallic state for zigzag $\mathrm{MoS}_{2}$ nanoribbon under a strain $s_{11}=4 \%$ and exchange coupling $h=\lambda_{M}$.

Figure 6. Variation of edge-state band gap with external strain for zigzag $\mathrm{MoS}_{2}$ nanoribbon with the width 4.1, 10 and $50 \mathrm{~nm}$. The dashed horizontal line indicates the zero value of band gap. 


\section{Figure}

(a)

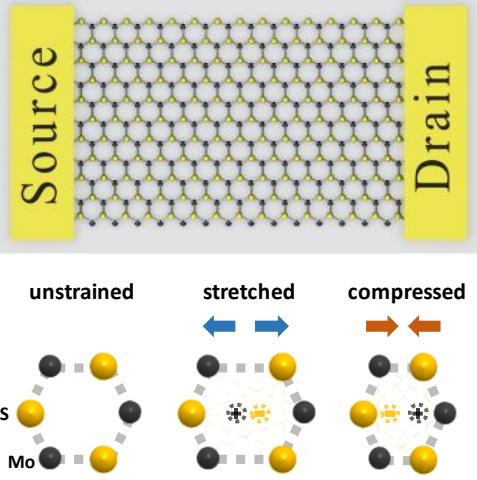

(b)

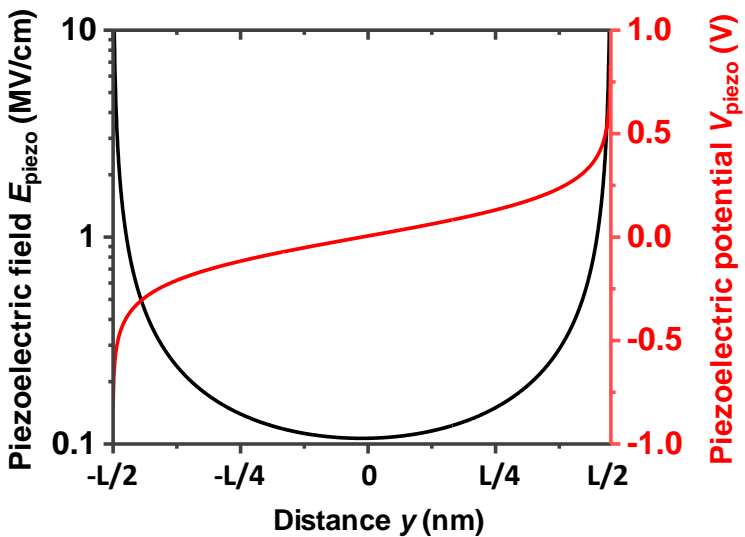

Figure 1 

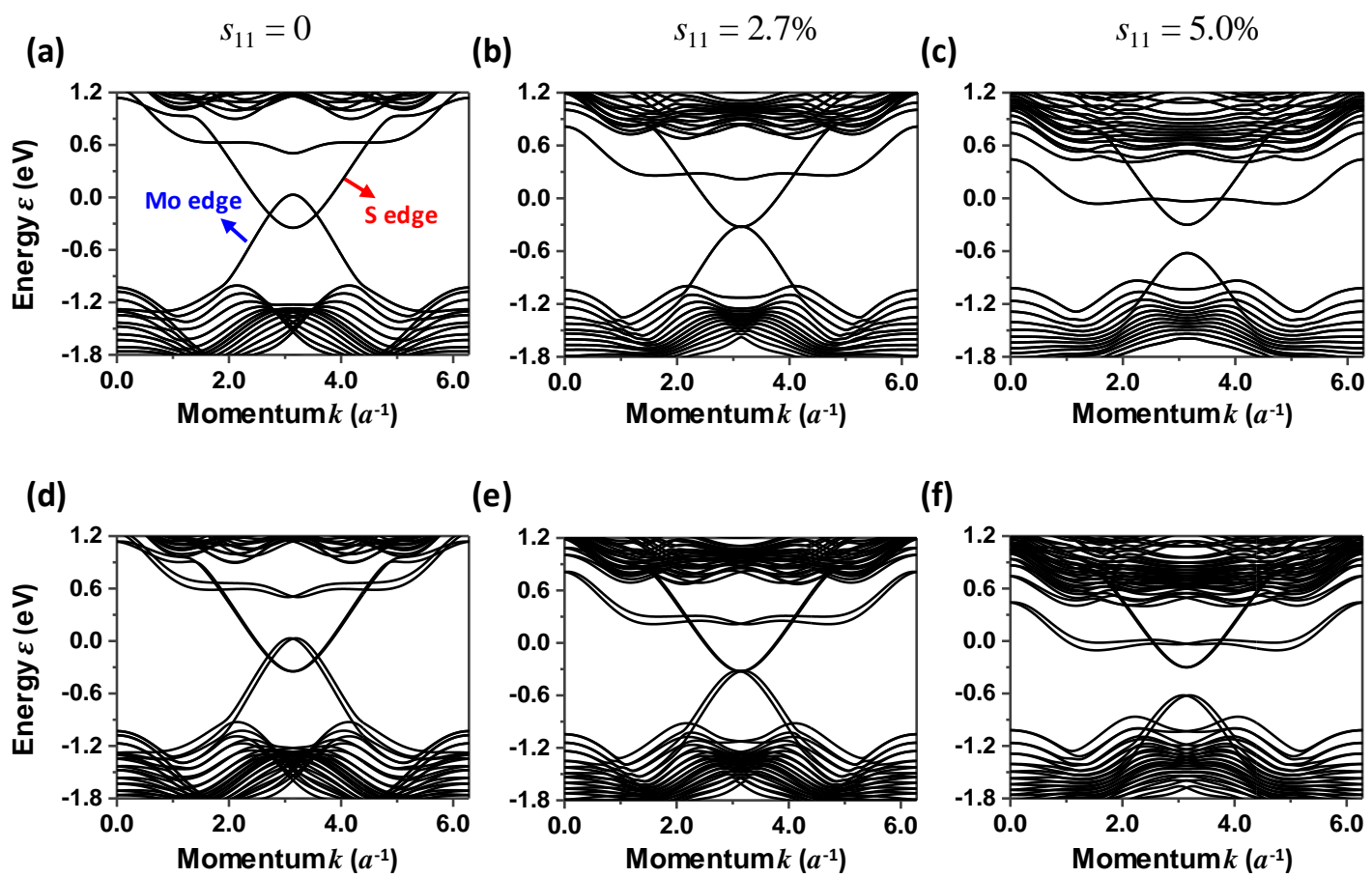

(e)

(f)
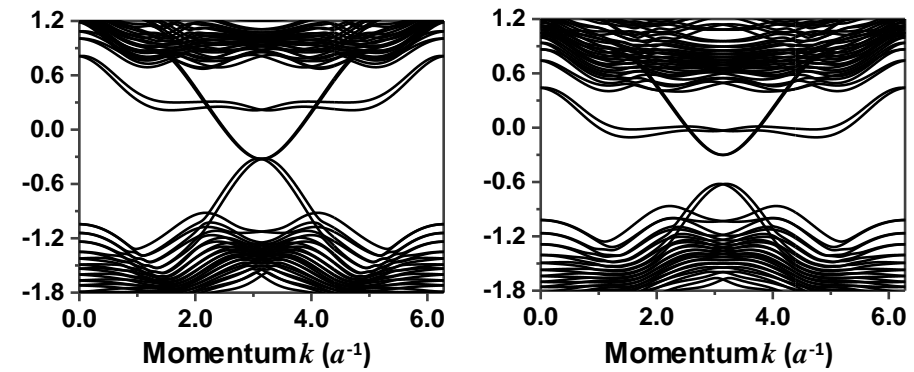

Figure 2 
(a)

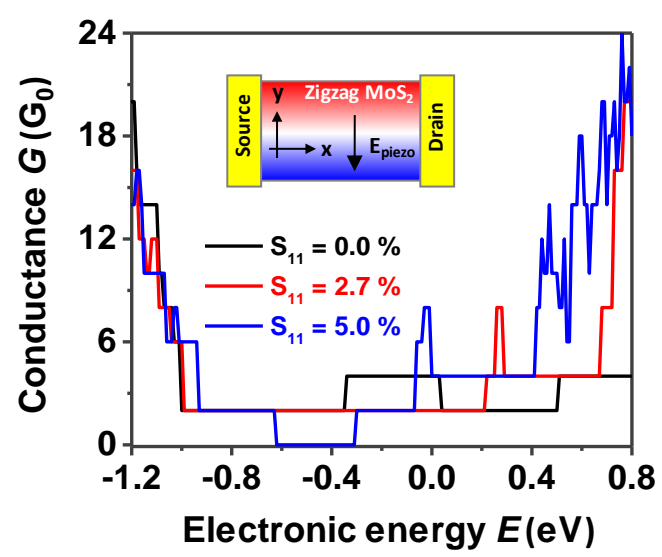

(b)

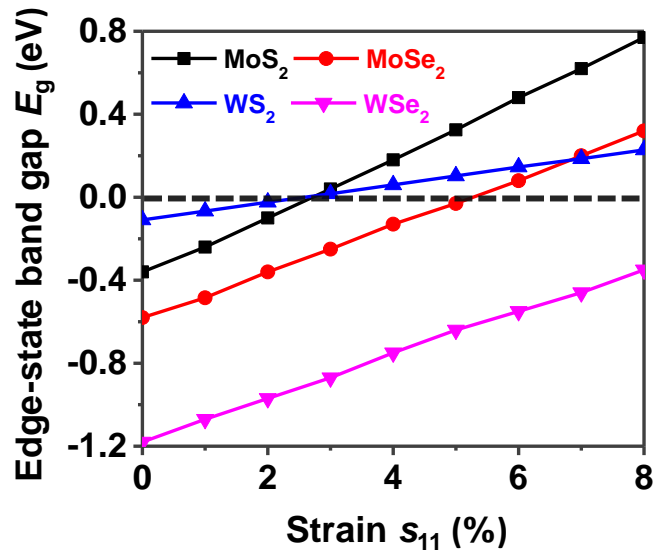

Figure 3 
(a)

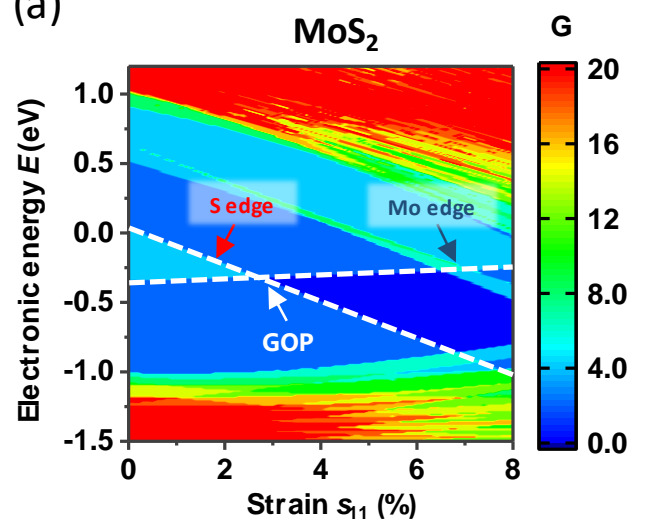

(c)

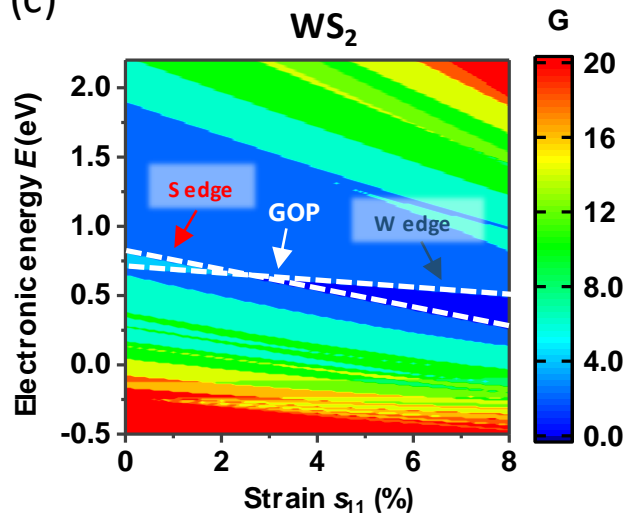

(b)

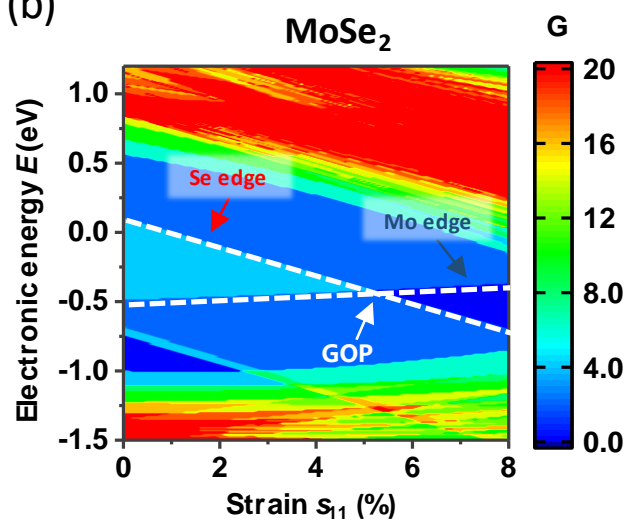

(d)

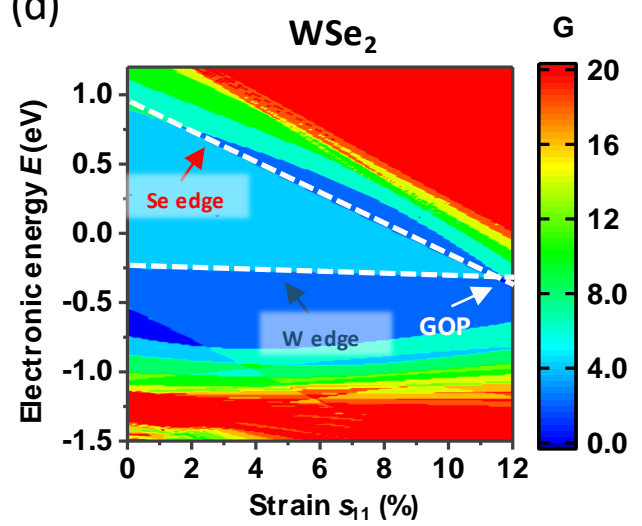

Figure 4 


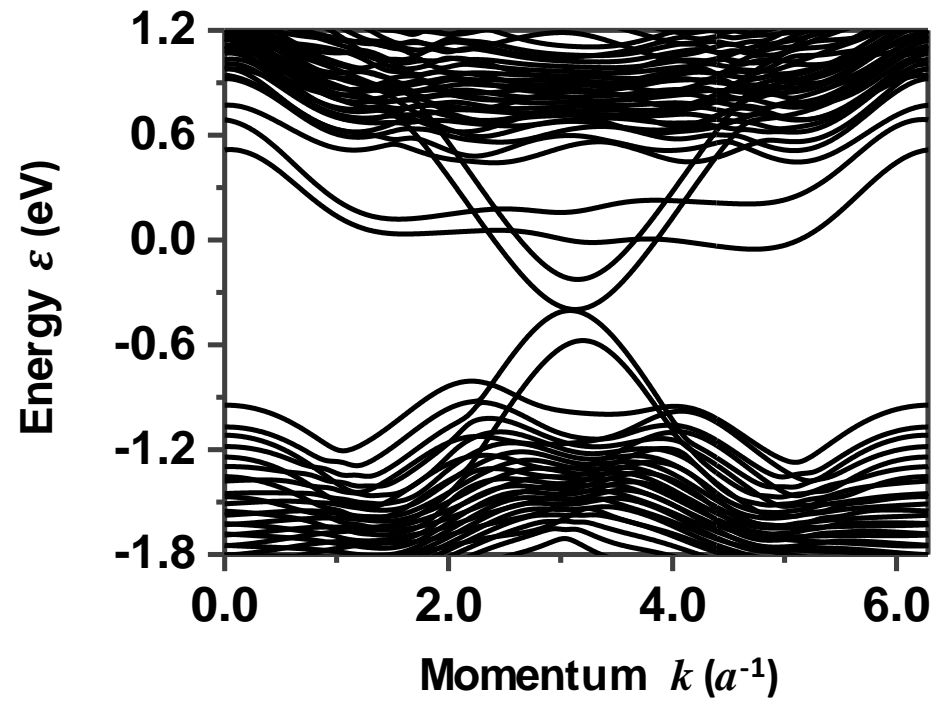

Figure 5 


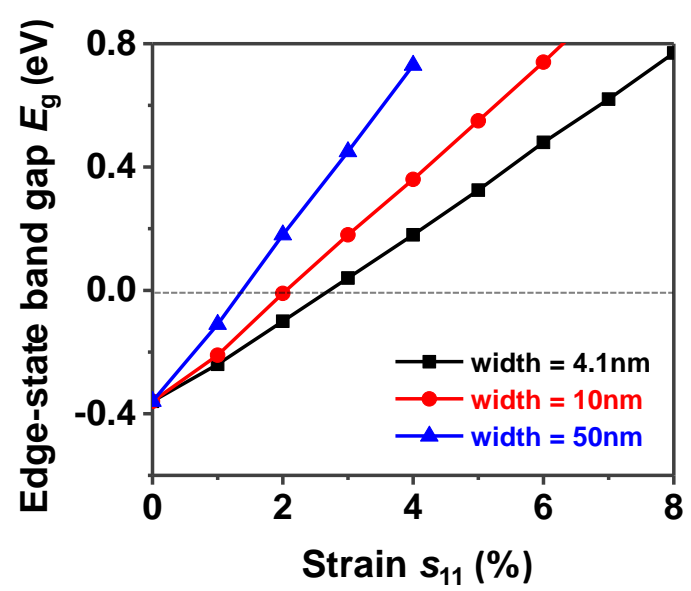

Figure 6 